\title{
Il castello templare di Peniscola, da fortezza di epoca crociata a cittadella tardo rinascimentale
}

\section{Stefano Bertocci}

DIDA-Dipartimento di Architettura, Università degli Studi di Firenze, Italia, stefano.bertocci@unifi.it

\begin{abstract}
The project of the digital survey of the fortress and the castle of Peniscola is born from a dual line of research, the study of the fortresses of the Crusader period in the Mediterranean basin and the research on the fortifications designed by Antonelli in the sixteenth and seventeenth century between Mediterrano and the Caribbean. Peniscola, first Greek and then Roman colony, in 718 A. D., it became part of the Muslim rule in the Iberian peninsula. These territories were under the control of the Moors until 1233, and between 1294 and 1307, the Knights of the Temple rebuilt the existing Muslim fortress. Important Mediterranean port and land border Peniscola, with the majestic ramparts designed in 1543, by order of Philip II, the engineer Giovanni Battista Antonelli, is also an important example of the new approach to the defense in connection with the expansion of 'use of firearms in the sixteenth century.
\end{abstract}

Keywords: Antonelli, digital survey, documentation, 3D laser scanning.

\section{Introduction}

Il progetto del rilievo della fortezza di Peniscola è nato da un duplice indirizzo di ricerche (lo studio delle fortezze di epoca crociata nel bacino Mediterraneo e la ricerca sulle fortificazioni progettate dagli Antonelli nei secoli XVI e VVII, architetti al servizio della Corona di Spagna fra Mediterraneo e Nuovo Mondo) che da anni si stanno sviluppando nel Laboratorio Congiunto interdipartimentale Landscape Survey \& Design, che ha sede nel Dipartimento di Architettura dell'Università di Firenze. Caratteristica del progetto di ricerca è la costruzione di banche dati affidabili, basate su aggiornate tecnologie di rilevamento digitale, delle fortificazioni coinvolte dalle ricerche in atto.

Peniscola, colonia prima greca e poi romana, dal 718 venne conquistata da Tarik ibn Zyad e divenne a tutti gli effetti parte del dominio mussulmano in Spagna. Questa città rimase sotto il controllo dei mori fino al 1233, anno in cui il re d'Aragona Giacomo I riuscì a riprendersi il possesso della città valenciana e la cedette all'Ordine Templare in cambio della città di Tortosa. Tra il 1294 e il 1307 i cavalieri dell'Ordine Templare riedificarono la preesistente fortezza musulmana. con tutti gli elementi che gli avrebbero conferito l'importante funzione di fortezza militare per il controllo di questa zona del Mediterraneo. Peniscola, colonia prima greca e poi romana, era un importante porto ed in periodo romano la città diventò un importante snodo di produzione manifatturiera di ceramiche, tessuti ed armi.

Nel 718 Tarik ibn Zyad, anche conosciuto come "il conquistatore" la città di che divenne parte del dominio mussulmano nella penisola Iberica. Questi territori rimasero sotto il controllo dei mori fino al 1233, anno in cui il re d'Aragona Giacomo I riuscì a riprendersi il possesso della città valenciana.

A partire dal 1294 i cavalieri dell'Ordine Templare riedificarono con un nuovo schema la preesistente forte zza musulmana loro affidata. 
Porto sul Mediterraneo e terra di confine Peniscola dalla fine del XIV secolo ad oggi ha implementato costantemente il suo sistema difensivo in funzione dell'importanza strategica che l'insediamento rivestiva. Il complesso del castello templare rimane ancor oggi pressoché intatto nella parte sommitale dell'insediamento, e si raggiunge attraversando la lingua di terra, oggi occupata da una parte dell'espansione moderna della cittadina, che congiunge il centro storico di Peniscola con la terraferma. Il castello, recentemente restaurato e destinato a museo, conservala sua antica imponenza come appare anche dalla documentazione storica ed iconografica, e consente dalle sue terrazze e dagli spalti, in accordo con la sua originaria funzione, un eccezionale controllo dal punto di vista panoramico su tutto il litorale.

Le modifiche apportate da Filippo II per armare la fortezza con l'artiglieria, così come i bombardamenti subiti durante le guerre e gli assedi, non hanno modificato sostanzialmente la forma del castello, che è stato dichiarato edificio nazionale nel 1931.

\subsection{Il castello templare di Peniscola, un esempio di architettura di epoca crociata in Spagna}

Il castello di Peniscola. venne costruito tra il 1294 e il 1307 alla maniera dei castelli templari, con tutti gli quegli elementi tipologici e strutturali, maturati in due secoli di esperienza costruttiva fra oriente ed occidente, richiesti dall'importante funzione militare. Nel 1307 Peniscola tornò sotto il controllo della corona e l'ordine Templare venne soppresso poco dopo. Tutti i suoi possedimenti passarono così al neonato ordine di Santa Maria di Montesa, che governò la città dal 1319 fino alla fine del XIV secolo.

Il rilievo del castello templare di Peniscola, condotto con tecnologia laser scanner nel 2011, in collaborazione con Digitarca s,n,c., ha permesso di accertare che la fortificazione, nelle sue parti maggiormente conservate, presenta una tipologia comune a molti castelli di origine crociata presenti, ad esempio, in Terrasanta, organizzati con due $\mathrm{o}$ più cinte di difese costituite da mura intervallate da torri di

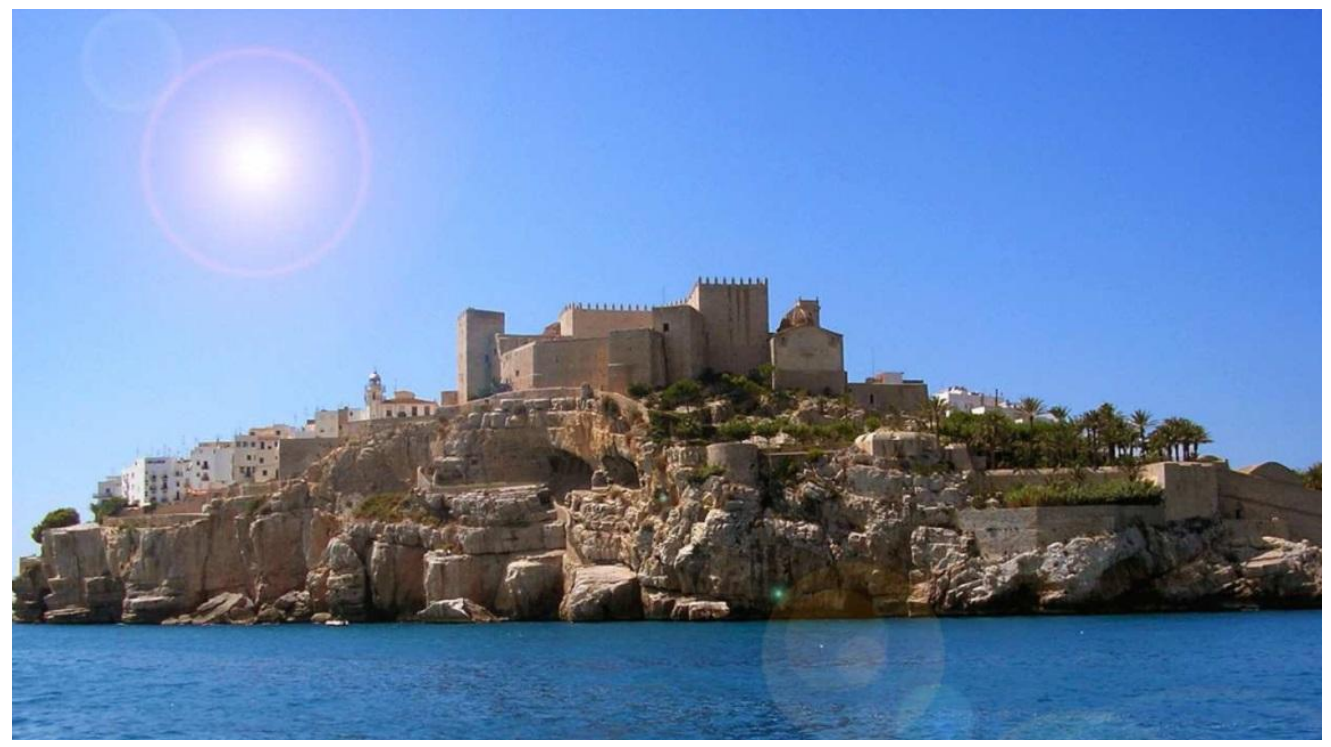

Fig. 1- Veduta di Peniscola dal Mare e del castello templare sopra la scogliera. 


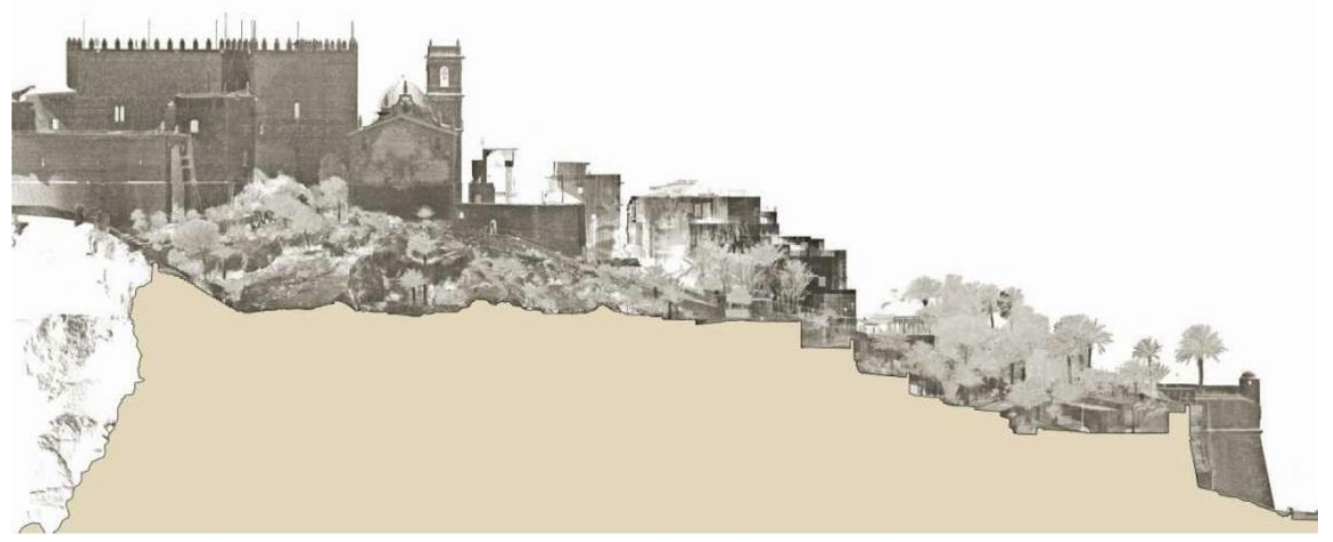

Fig. 2- Sezione del centro storico di Peniscola ottenuta dalla nuvola di punti laser scanner, nella quale sviene posto in relazione il volume delcastello con lo sviluppo dei sistemi difensivi rinascimentali.

controllo, con la parte alta della fortezza organizzata attorno ad una corte sulla quale si affacciano la grande chiesa ed il palazzo dell'Ordine Templare: queste strutture come accade anche nel più famoso Crak dei Cavalieri ad Oms in Siria, costituiscono con le loro possenti murature, parte della cinta più interna. Per rimanere nel territorio in esame esempi di riferimento sono presenti anche in Spagna, nel Regno di Valencia ed in Catalogna: si tratta di fortezze edificate spesso anche sui resti di insediamenti fortificati dai secoli precedenti, come il castello di Miravet (Tarragona), la rocca di Alcalà de Xivert (Castellò) ed il castello di Santa Magdalena de Pulpis (Castellò).

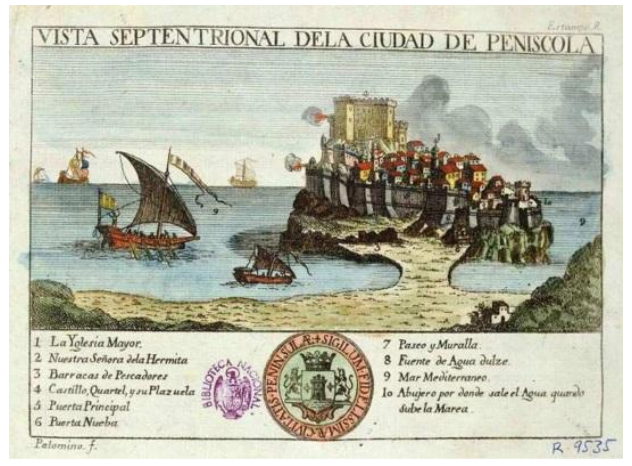

Fig. 3- Disegno di Peniscola del 1786.

Il castello di Peniscola faceva parte di una rete di fortificazioni allestite dai Cavalieri dell'Ordine Templare, lungo le maggiori vie di comunicazione dell'epoca, per assicurare il controllo di tutto il territorio. In particolare Peniscola, oltre a costituire un importante porto sul mediterraneo, si trova sulla via che collegava Miravet alla capitale del Regno, Valencia.

L'antica fortezza di Miravet è di origine islamica e venne ricostruita dall'Ordine dei Templari a metà del secolo XIII per il controllo della valle del fiume Ebro. L'impianto della fortificazione è quello comune agli sperimentati esempi della Terrasanta: una massiccia fortezza con una grande corte interna, con la cinta esterna rafforzata da torrioni a pianta rettangolare, edificata su più livelli sfruttando un rilievo roccioso ed un preesistente impianto fortificato. L'architettura e l'impianto ricordano, come accade spesso in questi casi le tipologie dei monasteri cistercensi con i vari locali disposti attorno al chiostro. Addossati alle mura i principali ambienti coperti con possenti volte a botte in pietra di ottima fattura: fra questi la grande cappella, con l'abside inglobato nello spessore delle cortine esterne, e la sala dei cavalieri o refettorio. Rimangono inoltre vari ambienti una volta destinati a tutte le funzioni del castello, magazzini, cantina, e cucina; si tratta infatti del secondo castello romanico più grande della Spagna.

La rocca di Xivert era una roccaforte islamica riferibile ai secoli X e XI che venne donata all'Ordine Templare da Alfonso II nel 1169; in seguito perduta venne definitivamente ripresa 
dai cavalieri nel 1233 e in buona parte ricostruita ed adeguata alle nuove esigenze militari.

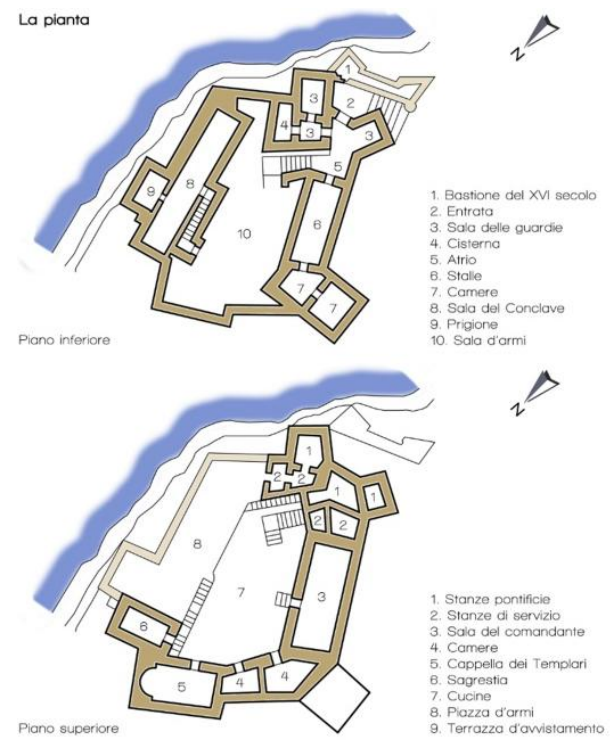

Fig. 4- Planimetrie del castello.

L'antica parte andalusa, in buona parte conservata, è esempio paradigmatico dello stile islamico e presenta murature in tapia. La riconversione ad opera dei Cavalieri Templari ha comportato il rinforzo di parte del recinto e un ripensamento generale degli ambienti. Le mura e le torri del castello dovevano mostrare il nuovo potere feudale dell'Ordine, così il lato nord fu ispessito e sopraelevato. Il settore meglio conservato è quello di levante, costituito da una cortina di $10 \mathrm{~m}$ di lunghezza fiancheggiata da due torri circolari. Sulla sommità del rilievo insiste l'ultima cinta fortificata con torri a pianta rettangolare e torri cilindriche che racchiude la grande corte con la cisterna.

Sul lato sud la chiesa, ad aula unica con il lato sud est facente parte del circuito murario, affiancata dal donjon e da una torre circolare dal lato absidale.

La fortezza di Pulpis, di origine islamica, è stata ceduta da Alfonso II all'Ordine Templare nel 1190. Situata tra Peniscola e Alcalà de Chivert, si alza sopra un'altura aspra e rocciosa della Sierra de Irta. Controllava l'antico cammino da Tortosa a Valencia. Poggia su una piattaforma rotondeggiante con una punta sul lato sud. La porzione maggiormente inespugnabile risulta essere quella che va da sud-est a sud-ovest, a causa della parete molto scoscesa, mentre il pendio nord è più dolce e accessibile. E' qui che troviamo le maggiori opere di fortificazione, per la maggiore vulnerabilità dell'accesso.

Sotto Benedetto XIII furono eseguiti alcuni lavori per la trasformazione dell'arcigna fortezza in un palazzo papale.

Le pareti sono in pietra lavorata e tutte le camere sono voltate a botte, che si innalzano da conci molto semplici. Gli incavi delle porte sono formati da archi con grosse pietre a cuneo.
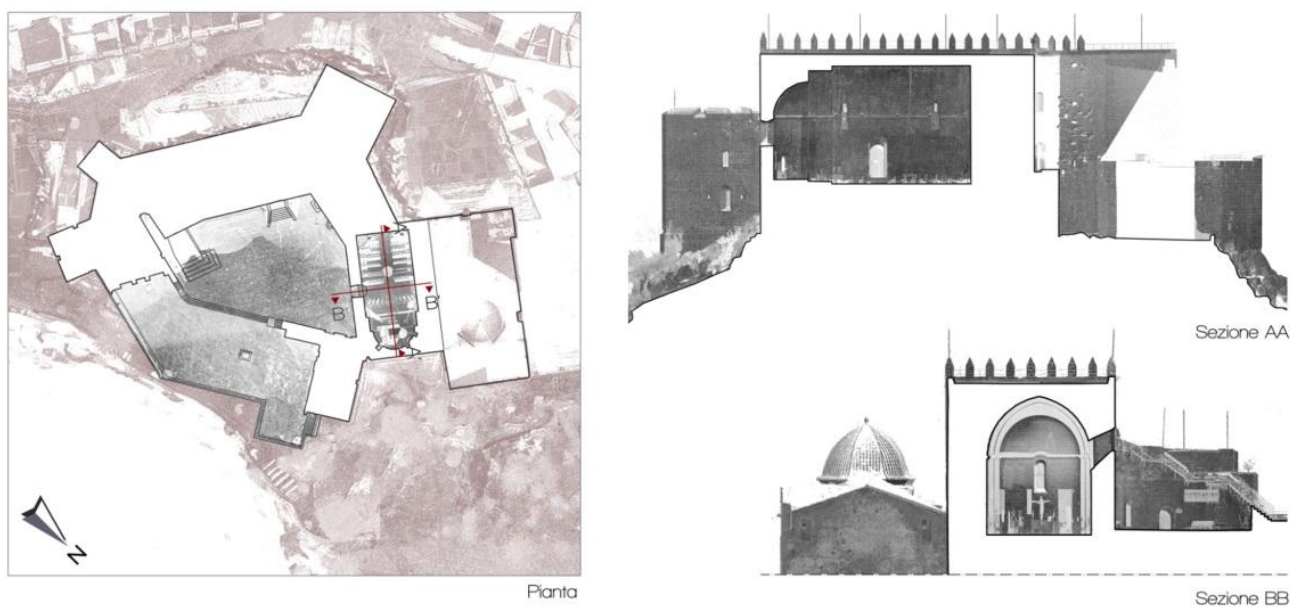

Fig. 5- Sezioni della chiesa templare ottenute dalla nuvola di punti laser scanner. 
Gli ambienti più interessanti del castello sono il "Corpo delle Guardie" e la Chiesa dei Templari, usata da Benedetto XIII e Clemente VIII come basilica papale. Con le trasformazioni avvenute durante il periodo dello Scisma d'Occidente, quando divenne residenza del Papa Benedetto XIII di Avignone, conosciuto anche come Papa Luna, e gli ampliamenti con la maestosa cinta bastionata progettata nel 1543, per ordine di Filippo II, dall'ingegnere Giovanni Battista Antonelli, Peniscola resistette a numerosi assedi subiti, dai primi per opera degli anglo-olandesi agli inizi del XVIII secolo a quelli, cento anni più tardi, condotti dalle truppe di Napoleone.

La città di Peniscola perse la funzione di fortezza solo nel 1890.

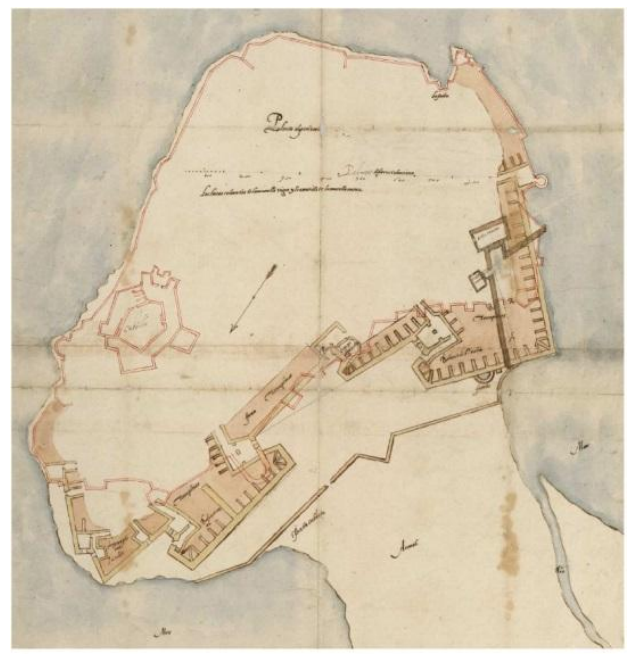

Fig. 6- Progetto per l'ampliamento della muraglia difensiva del 1579. Archivio General de Simancas, Mapas Dibujos y Planos: 09.059.

\section{Il rilievo e la documentazione della cinta bastionata di Peniscola}

Il rilievo delle mura di Peniscola si inserisce nel quadro della ricerca sugli Antonelli, architetti ed ingegneri militari al servizio della corona di Spagna fra XVI e XVII secolo nel Mediterraneo e nelle Americhe.

Lo studio riguarda la documentazione dei corpi di fabbrica che, imponenti, connotano l'immagine dell'intero complesso urbano e, attraverso l'utilizzo di strumentazione laser scanner, vengono analizzate le forme del costruito con lo scopo di determinare modelli utili alla gestione dell'impianto urbano ed alla comprensione delle architetture che caratterizzano il sistema difensivo, anche in un'ottica di valorizzazione delle stesse opere. $\mathrm{La}$ trasformazione che ha interessato lo sviluppo urbano di Peniscola dalla fine del XIV secolo ad oggi ha riguardato costantemente l'ampliamento del sistema difensivo in funzione dell'importanza strategica che l'insediamento rivestiva.

Dalle trasformazioni avvenute durante il periodo dello Scisma d'Occidente, quando Papa Benedetto XIII di Avignone, conosciuto anche come Papa Luna, riuscì a togliere il castello ai possedimenti dell'ordine di Santa Maria di Montesa, ai numerosi assedi subiti, dai primi per opera degli anglo-olandesi agli inizi del XVIII secolo a quelli, cento anni più tardi, condotti dalle truppe di Napoleone, la città di Peniscola perse la funzione di fortezza solo nel 1890.

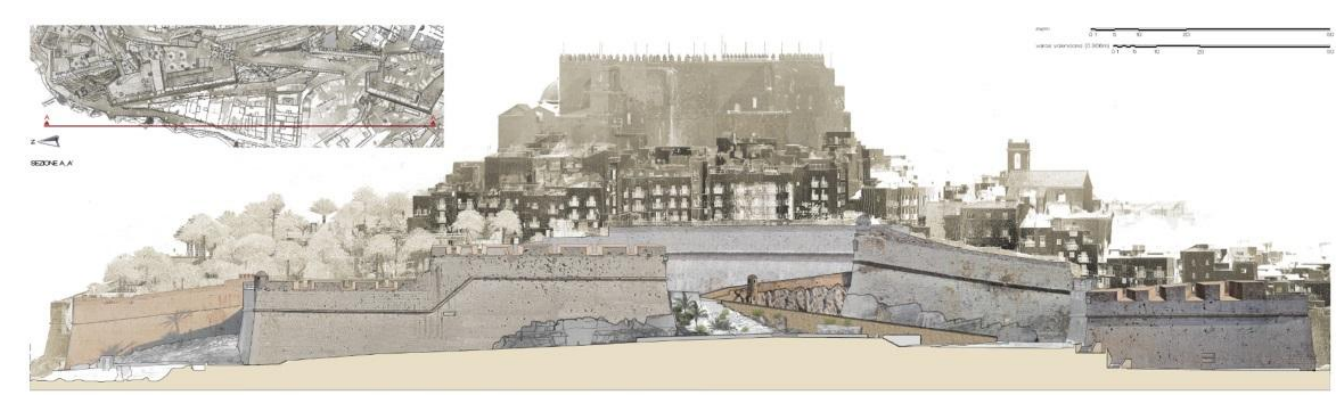

Fig. 7- Prospetto del centro storico di Peniscola dal versante di terra. 
Se l'importanza militare della città aveva segnato il suo sviluppo fino alla fine del XIX secolo, furono gli eventi socio-economici del secolo successivo a lasciare fortemente il segno e a cambiare completamente l'aspetto della città. Il centro di Peniscola, importante meta turistica della costa spagnola, è tuttavia denso di maestose opere difensive che custodiscono, nelle geometrie e nei modelli compositivi adottati, importanti informazioni che riguardano le tecnologie le tecniche costruttive delle architetture militari alla moderna.

\section{References}

AA.VV. (1999). A journey through history, Patronato Municipal de Turismo de Peniscola. $1^{\text {a }}$ Edición.

Balaguer Dezcallar P. (2012). "La restauracion de las fortificaciones de Felipe II"., in Atti del IV

Balaguer Vicen I. (2012). Restauración y consolidación de las murallas de Peñíscola (Castellón), Ministerio de Educación, Cultura y Deporte - IPCE, Madrid.

Bertocci S. Parrinello S. (2015). Digital Survey and Documentation of the Archaeological and Architectural sites UNESCO World Heritage List. Edifir, Edizioni. Firenze.

Cassi Ramelli A., (1995). Dalle caverne ai rifugi blindati. Casa editrice Adda, Milano

Congreso de Castellologia, Madrid, 7 - 9 Marzo 2012, pp. 1173-1191.

Fuguet Sans J. (2009). Templers i hospitalers II. Guia de les terres de l'ebre i castells templers del baix maestrat. Rafael Dalmau Editor. Barcellona

Simò Castillo J. B. (2002). Visitar y conocer peñiscola. Editorial Antinea. Valencia. 